\title{
Hard X-ray emission clumps in the $\gamma$-Cygni supernova remnant: An INTEGRAL-ISGRI view
}

\author{
A. M. Bykov ${ }^{1}$, A. M. Krassilchtchikov ${ }^{1}$, Yu. A. Uvarov $^{1}$, H. Bloemen ${ }^{2}$, R. A. Chevalier ${ }^{3}$, \\ M. Yu. Gustov ${ }^{1}$, W. Hermsen ${ }^{2}$, F. Lebrun ${ }^{4}$, T. A. Lozinskaya ${ }^{5}$, G. Rauw ${ }^{6}$, T. V. Smirnova ${ }^{7}$, \\ S. J. Sturner ${ }^{8}$, J.-P. Swings ${ }^{6}$, R. Terrier ${ }^{4}$, and I. N. Toptygin ${ }^{1}$
}

\begin{abstract}
${ }^{1}$ A. F. Ioffe Institute for Physics and Technology, 26 Polytechnicheskaia, 194021 St. Petersburg, Russia e-mail: byk@astro.ioffe.ru

2 SRON National Institute for Space Research, Sorbonnelaan 2, 3584 CA Utrecht, The Netherlands

3 Department of Astronomy, University of Virginia, PO Box 3818, Charlottesville, VA 22903, USA

${ }^{4}$ CEA - Saclay, DSM/DAPNIA/Service d'Astrophysique, 91191 Gif-sur-Yvette Cedex, France

5 Sternberg Astronomical Institute, Moscow State University, 13 Universitetskij, 119899 Moscow, Russia

${ }^{6}$ Institut d'Astrophysique et de Géophysique, Université de Liège, Allée du 6 Août 17, Bât. B5c, 4000 Liège, Belgium

7 Astro Space Center of the Lebedev Physics Institute, 84/32 Profsoyuznaia, 117810 Moscow, Russia

8 NASA Goddard Space Flight Center, Code 661, Greenbelt, MD 20771, USA
\end{abstract}

Received 22 September 2004 / Accepted 9 October 2004

\begin{abstract}
Spatially resolved images of the galactic supernova remnant G78.2+2.1 ( $\gamma$-Cygni) in hard X-ray energy bands from $25 \mathrm{keV}$ to $120 \mathrm{keV}$ are obtained with the IBIS-ISGRI imager aboard the International Gamma-Ray Astrophysics Laboratory INTEGRAL. The images are dominated by localized clumps of about ten arcmin in size. The flux of the most prominent NorthWestern $(\mathrm{NW})$ clump is $(1.7 \pm 0.4) \times 10^{-11} \mathrm{erg} \mathrm{cm}^{-2} \mathrm{~s}^{-1}$ in the $25-40 \mathrm{keV}$ band. The observed X-ray fluxes are in agreement with extrapolations of soft X-ray imaging observations of $\gamma$-Cyg by ASCA GIS and spatially unresolved RXTE PCA data. The positions of the hard X-ray clumps correlate with bright patches of optical line emission, possibly indicating the presence of radiative shock waves in a shocked cloud. The observed spatial structure and spectra are consistent with model predictions of hard X-ray emission from nonthermal electrons accelerated by a radiative shock in a supernova interacting with an interstellar cloud, but the powerful stellar wind of the O9V star HD 193322 is a plausible candidate for the NW source as well.
\end{abstract}

Key words. gamma rays: observations $-\mathrm{X}$ rays: ISM: supernova remnants - individual: $\mathrm{G} 78.2+2.1(\gamma$-Cygni $)-$ radiation mechanisms: nonthermal - cosmic rays

\section{Introduction}

The supernova remnant (SNR) G78.2+2.1 ( $\gamma$-Cygni) is a degree size extended source which has been imaged in radio waves to $\gamma$-rays. The SNR is located in the complex Cygnus X region of massive gas and dust complexes and close to the most powerful Cyg OB2 association. Radio observations by Higgs et al. (1977) established a shell-like structure of the remnant, suggested also by Lozinskaya (1977). Multi-frequency radio observations by Zhang et al. (1997) revealed a patchy structure of the radio spectral-index distribution. The integrated radio spectral index $\alpha=0.54 \pm 0.02$, with variations of \pm 0.15 within the remnant. Prominent radio brightness enhancements are present in the South-East and the North-West of $\gamma$-Cyg (e.g. Zhang et al. 1997). A distance to $\gamma$-Cyg of $\sim 1.5 \mathrm{kpc}$ was estimated by Landecker et al. (1980) from radio HI observations.

Optical images of $\gamma$-Cyg in $\mathrm{H}_{\alpha}+[\mathrm{NII}]$, [SII] and [OIII] filters, recently presented by Mavromatakis (2003), clearly show a patchy structure with bright spots of some ten arcminutes scale and line emission fluxes of a few times $10^{-15} \mathrm{erg} \mathrm{cm}^{-2} \mathrm{~s}^{-1} \operatorname{arcsec}^{-2}$ for $\mathrm{H}_{\alpha}$ filters and a few times $10^{-16} \mathrm{erg} \mathrm{cm}^{-2} \mathrm{~s}^{-1} \operatorname{arcsec}^{-2}$ for the [OIII] filter.

Archival ROSAT and ASCA observations of G78.2+2.1 were analyzed by Lozinskaya et al. (2000). They pointed out a complex structure of the remnant with ROSAT emission extending well beyond the apparent SNR radio shell, possibly indicating expansion into a progenitor star wind cavity. With archival ASCA observations, Uchiyama et al. (2002) found X-ray emission above $4 \mathrm{keV}$ to be dominated by several localized clumps, mostly in the Northern part of the remnant. The $4-10 \mathrm{keV}$ emission of the clumps has a hard photon index of 0.8-1.5.

A high-energy $\gamma$-ray source, 2CG $078+2$, was discovered in the field of $\gamma$-Cyg with the $\operatorname{COS} B$ satellite (e.g. Swanenburg et al. 1981). CGRO-EGRET confirmed this source (2EG J2020+4026/3EG J2020+4017), which is one of the brightest steady-state unidentified sources in the EGRET catalogue with a flux of $\sim 1.2 \times 10^{-6} \mathrm{~cm}^{-2} \mathrm{~s}^{-1}$ above $100 \mathrm{MeV}$ (Sturner \& Dermer 1995; Esposito et al. 1996). Whipple $\gamma$-ray 


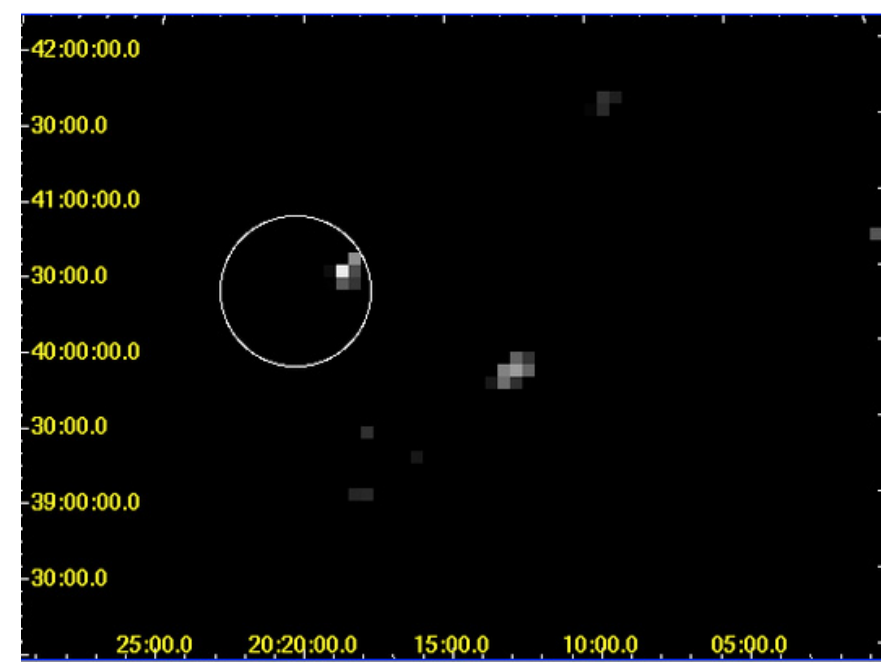

Fig. 1. The ISGRI 25-40 keV broad view of the $\gamma$-Cyg region (linearly scaled $\mathrm{S} / \mathrm{N}$ map of the sources above $2 \sigma$ ). The solid circle is the same as in Fig. 2.

telescope observations (e.g. Buckley et al. 1998) established an upper limit of $2.2 \times 10^{-11} \mathrm{~cm}^{-2} \mathrm{~s}^{-1}$ for the flux above $300 \mathrm{GeV}$, indicating a break in the high-energy emission spectrum above a few GeV. The $\gamma$-ray emission might be produced by a pulsar, but can be attributed to interactions of accelerated energetic particles with ambient matter and radiation as well (e.g. Sturner et al. 1997; Gaisser et al. 1998; Bykov et al. 2000). Solving this problem of the origin of the $\gamma$-ray emission requires the analysis of multiwavelength imaging observations. Hard X-ray imaging provides a crucial tool to distinguish between leptonic and hadronic contributions to the $\gamma$-ray emission. We present below the first hard X-ray imaging observation of $\gamma$-Cyg.

\section{Observations and data analysis}

\subsection{INTEGRAL ISGRI observations}

The SNR G78.2+2.1 was observed with the ISGRI imager (Lebrun et al. 2003) aboard INTEGRAL (Winkler et al. 2003). We combined data from 21 Core Program and calibration observations (revolutions 12-82: Nov. 18, 2002-Jun. 15, 2003) and added 28 pointings from AO1 (rev. 80, 11-12 Jun. 2003) and AO2 (rev. 191, 8-9 May 2004) observations (Obs. Ids 0129700 and 0229700: amalgamated observations with G. Rauw as PI).

The data obtained from ISGRI have been reduced with the standard off-line scientific analysis software developed at the INTEGRAL Science Data Center (the OSA 4.0 package). The standard good time selection criteria were applied; only science windows (SCWs) with more than $100 \mathrm{~s}$ of good time were considered. Wide energy bands were used (25$40 \mathrm{keV}, 40-80 \mathrm{keV}$, and $80-120 \mathrm{keV}$ ) in order to be able to detect sources at a few mCrab level. In order to improve on source detection (at the cost of source-localization accuracy), pixel spreading was switched off. We analysed 360 good ks of FCFOV (fully coded field of view) observations and 1060 good ks of PCFOV (partially coded field of view) observations, although only FCFOV data were used for the flux estimates presented here. Source reconstruction with FCFOV data is more secure in the Cygnus region with its bright point sources and complex diffuse background emission. The angular resolution (FWHM) of ISGRI is about $12^{\prime}$ and the images were sampled in $\sim 5^{\prime}$ pixels (Lebrun et al. 2003; Goldwurm et al. 2003).

Source significances were derived from a statistical distribution of count-rate values for a sample of about $100 \times 100$ pixels in ISGRI FCFOV images around $\gamma$-Cyg (using the mean and the standard deviation of the distribution).

At present, the most reliable ISGRI flux estimates can be obtained by cross calibration with the Crab, i.e. by comparing the source and Crab count rates in the same energy bands, accounting for different [model] spectral distributions. The systematic uncertainties of this procedure are estimated to be about $25 \%$, mainly due to the possibly extended nature $\left(z 10^{\prime}\right)$ of the detected sources (cf. Lubinski 2004, where calibrations of point sources are discussed).

In Figs. 1 and 2 we show X-ray images of G78.2+2.1 obtained with INTEGRAL ISGRI, ASCA, and ROSAT. The ISGRI images in our three energy bands are dominated by a few localized clumps of emission. The flux estimates of the NorthWestern $(\mathrm{NW})$ clump are $(1.7 \pm 0.4) \times 10^{-11} \mathrm{erg} \mathrm{cm}^{-2} \mathrm{~s}^{-1}$ in the $25-40 \mathrm{keV}$ band, $(1.2 \pm 0.8) \times 10^{-11} \mathrm{erg} \mathrm{cm}^{-2} \mathrm{~s}^{-1}$ in the $40-80 \mathrm{keV}$ band, and $(2.5 \pm 1.2) \times 10^{-11} \mathrm{erg} \mathrm{cm}^{-2} \mathrm{~s}^{-1}$ in the $80-120 \mathrm{keV}$ band. For the SE clump we find $(1.2 \pm 0.5) \times 10^{-11} \mathrm{erg} \mathrm{cm} \mathrm{cm}^{-2} \mathrm{~s}^{-1}(25-40 \mathrm{keV}),(1.5 \pm$ $0.8) \times 10^{-11} \mathrm{erg} \mathrm{cm}^{-2} \mathrm{~s}^{-1}(40-80 \mathrm{keV})$, and $(3.0 \pm 1.2) \times$ $10^{-11} \mathrm{erg} \mathrm{cm}^{-2} \mathrm{~s}^{-1}(80-120 \mathrm{keV})$. The NE clump that coincides with the ASCA hard source $\mathrm{C} 1$ (Uchiyama et al. 2002) is seen only in the $40-80 \mathrm{keV}$ band with a flux of $(1.7 \pm 0.7) \times$ $10^{-11} \mathrm{erg} \mathrm{cm}^{-2} \mathrm{~s}^{-1}$. A longer exposure with ISGRI is required to detect (or reject) this NE-clump emission; the weak detection obtained so far might be related to the extended nature of the ASCA C1 source.

\subsection{RXTE PCA and ASCA GIS observations}

Hard X-ray emission from G78.2+2.1 can be also constrained from non-imaging $R X T E$ observations. We used public archive RXTE $61.4 \mathrm{ks}$ observations of $\gamma$-Cyg performed on 5-10 Apr. 1997. The RXTE PCA data were reduced with the standard HEASARC FTOOLS 5.3 software. Only Standard-2 mode data were used. The PCA background was accounted for with the latest faint source background model (cmfaintl7_eMv20031123). The standard good time selection procedure was applied. The RXTE PCA field of view covers a substantial part of $\gamma$-Cyg including the hard clumps seen with INTEGRAL ISGRI. We found the X-ray emission of $\gamma$-Cyg to extend beyond $\sim 20 \mathrm{keV}$ and fitted the RXTE PCA 5-15 keV data simultaneously with archival ASCA GIS2 3-7 keV data for the total remnant emission with a broken power-law with a Lorentz line at $6.2 \mathrm{keV}$. Here we used the same set of ASCA GIS data that was analyzed by Uchiyama et al. (2002).

The total remnant emission $(3-15 \mathrm{keV})$ was fitted by a power-law with photon indexes $\alpha_{1}=2.0 \pm 0.4$ below the break energy $E_{\mathrm{b}}=11.1 \pm 1.2 \mathrm{keV}$ and $\alpha_{2}=1.2 \pm$ 0.4 for $E_{\mathrm{b}}<E<15 \mathrm{keV}$ (the reduced $\chi_{v}^{2}=0.91$ at 

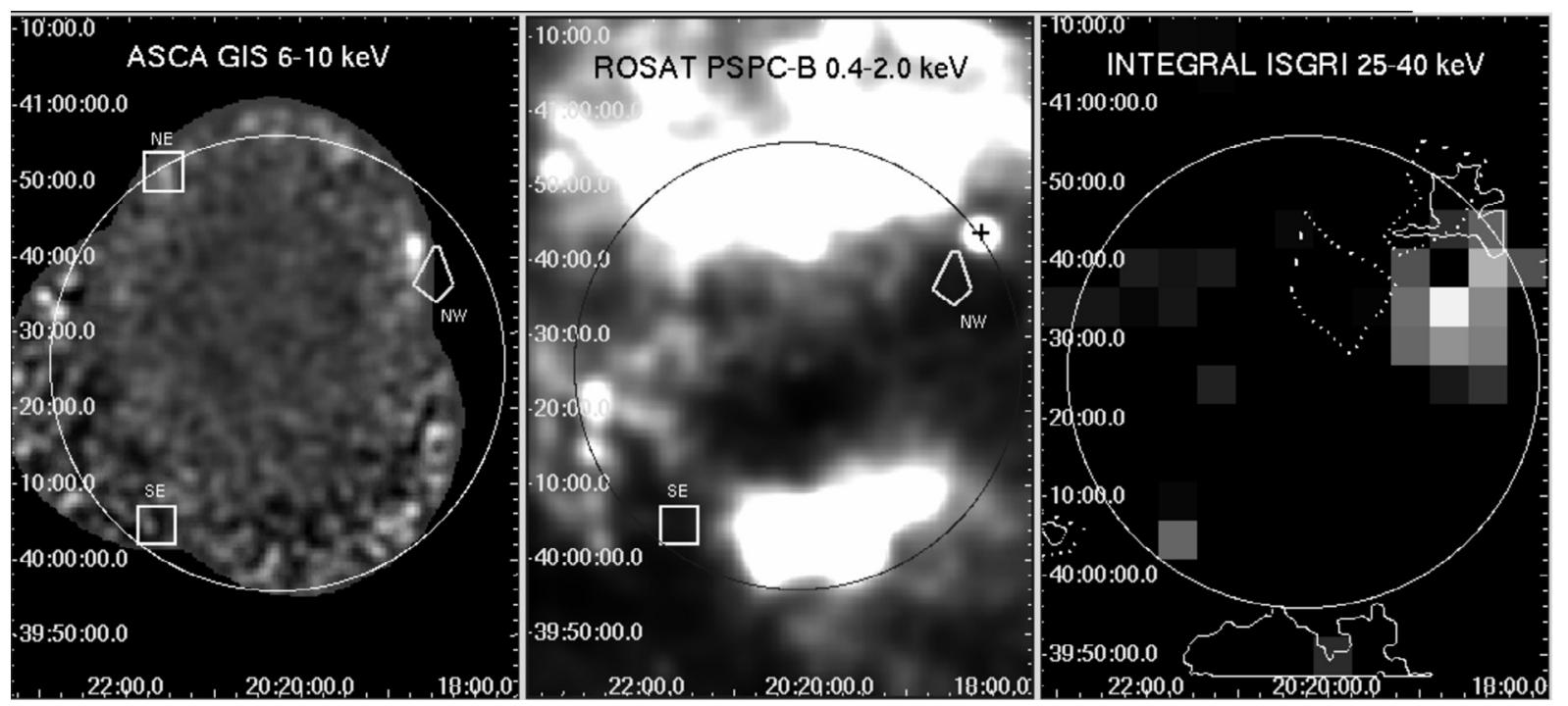

Fig. 2. Left: ASCA GIS 6-10 keV grayscale map with an INTEGRAL ISGRI 25-40 keV 4.2 $\sigma$ contour in the NW area. Centre: ROSAT PSPC-B 0.4-2.0 keV grayscale map with the same INTEGRAL ISGRI 25-40 keV 4.2 $\sigma$ contour. Right: INTEGRAL ISGRI 25-40 keV significance map (linear scale) with $\mathrm{H}_{\alpha}+[\mathrm{NII}] 6560 \AA 3 \times 10^{-15} \mathrm{erg} \mathrm{cm}^{-2} \mathrm{~s}^{-1} \operatorname{arcsec}^{-1}$ contours (dotted line) and [OIII] $5010 \AA 3 \times 10^{-16} \mathrm{erg} \mathrm{cm}^{-2} \mathrm{~s}^{-1} \mathrm{arcsec}^{-1}$ contours (solid line) (optical data are from Mavromatakis 2003). The hard emission regions NW, NE, and SE are marked in the first two panels. The NE clump is seen with ISGRI only in the $40-80 \mathrm{keV}$ band. The RXTE PCA field of view is indicated by the large solid circle. The black cross on the ROSAT map marks the position of the O9V star HD 193322 (20:18:07, +40:43:55). All maps are made for J2000.

$v=85$ d.o.f.). The power-law normalization was $(8.1 \pm 0.56) \times$ $10^{-3} \mathrm{ph} \mathrm{cm}^{-2} \mathrm{~s}^{-1} \mathrm{keV}^{-1}$ at $1 \mathrm{keV}$. We found a correction factor $(0.72 \pm 0.19)$ for the RXTE PCA flux normalization considered as a free parameter with a fixed ASCA GIS2 flux normalization. The line at $6.2 \pm 0.04 \mathrm{keV}$ has a width of $1.0 \pm 0.2 \mathrm{keV}$ (equivalent width is $1.1 \pm 0.5 \mathrm{keV})$ and normalization $(2.5 \pm$ $0.8) \times 10^{-4} \mathrm{ph} \mathrm{cm}^{-2} \mathrm{~s}^{-1}$. Note here that a similar signature of a $6.4 \mathrm{keV}$ line was found by Pannuti et al. (2003) in the RXTE analysis of another extended SNR G347.3-0.5. While the low energy branch of the fit is a combination of thermal and nonthermal components, the high energy emission above the break is nonthermal. Using the joint ASCA GIS2 - RXTE $P C A$ fit, we estimated the ranges of the extrapolated hard X-ray fluxes of the total remnant emission in the $25-40 \mathrm{keV}$ band as $(1.0-1.7) \times 10^{-11} \mathrm{erg} \mathrm{cm}^{-2} \mathrm{~s}^{-1}$, while in the $80-120 \mathrm{keV}$ regime the range is $(1.4-4.5) \times 10^{-11} \mathrm{erg} \mathrm{cm}^{-2} \mathrm{~s}^{-1}$. Since the hard X-ray ISGRI images are dominated by the NW, NE, and SE clumps that are located at the boundary of the RXTE PCA pointing, we have applied flux corrections accounting for the RXTE PCA collimator response at $\sim 30^{\prime}$ from the axis.

The total flux estimations are in agreement with the summed INTEGRAL ISGRI fluxes of the NW, NE, and SE clumps if a $25 \%$ systematic error in the calibration procedure is accounted for. The hard-emission spectrum of $\gamma$-Cyg is presented in Fig. 3. We show the total remnant ASCA GIS2 - RXTE $P C A$ flux, the fluxes of the ISGRI NW clump and of EGRET 3EG J2020+4017 source, and the Whipple upper limit.

\section{Discussion}

The locations of the hard X-ray clumps detected by INTEGRAL ISGRI correlate with dim regions of high $N_{\mathrm{H}} \gtrsim 5 \times 10^{21} \mathrm{~cm}^{-2}$ in the ROSAT PSPC-B $0.4-2.0 \mathrm{keV}$ greyscale map in Fig. 2.

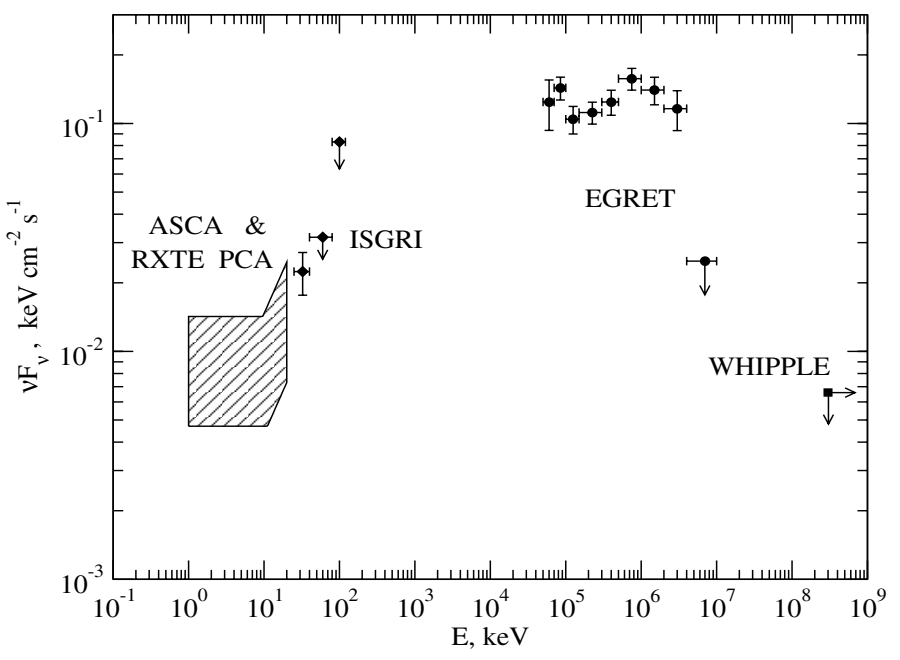

Fig. 3. The spectral energy distribution of the hard emission from $\gamma$-Cyg. The ISGRI datapoints correspond to the NW clump only.

The clumps adjoin the patches of bright optical $\mathrm{H}_{\alpha}+[\mathrm{NII}]$, [SII] and [OIII] line emission (of $\gtrsim 10^{\prime}$ scale size) observed by Mavromatakis (2003). The line emission may indicate an interaction of the SNR with a nearby cloud and the presence of a radiative shock wave.

In evolved supernova remnants interacting with interstellar clouds, such as $\gamma$-Cyg and IC 443, a highly inhomogeneous structure consisting of a forward shock of a moderate Mach number, a cooling layer, a dense radiative shell, and an interior region filled with hot tenuous plasma is expected. A SNR evolving in the inter-clump medium of a molecular cloud of density $n_{a} \sim 25 \mathrm{H}$ atoms $\mathrm{cm}^{-3}$ becomes radiative at radii $\sim 6 \mathrm{pc}$ (Chevalier 1999). A model of nonthermal electron acceleration and interactions in such SNRs by 
Bykov et al. (2000) predicted that these SNRs are efficient electron accelerators and sources of hard X- and $\gamma$-ray emission. A distinctive feature of the model is the presence of emitting clumps with very flat hard X-ray spectra of photon indexes $\alpha \sim 1$. The photon break energy and the maximum energy of accelerated electrons depend on the ambient density. A radiative shock propagating through the interclump medium could accelerate radio-emitting electrons in the $\mathrm{GeV}$ regime, also producing hard X-ray and $\gamma$-ray EGRET-regime emission. A slower MHD shock in a dense molecular clump could produce bright hard X-ray emission without prominent radio and $\gamma$-ray counterparts. Bremsstrahlung radiation from the nonthermal electrons has a hard X-ray photon spectrum. The nonthermal X-ray flux $F_{\mathrm{x}}$ from the electrons accelerated by a MHD shock of a velocity $v_{\mathrm{s} 7}$ (in $100 \mathrm{~km} \mathrm{~s}^{-1}$ ) and of an apparent angular size $\theta$ (in arcmin) can be estimated as a fraction of the shock ram pressure:

$$
F_{\mathrm{x}} \approx 9.2 \times 10^{-12} \eta \frac{n_{a} v_{\mathrm{s} 7}^{3}}{2} \theta^{2} \quad \mathrm{erg} \mathrm{cm}^{-2} \mathrm{~s}^{-1}
$$

The efficiency of X-ray bremsstrahlung emission $\eta$ is relatively low due to the Coulomb losses of fast particles, providing powerful optical and IR diffuse emission from the interclump medium. Having in mind that $\eta<10^{-5}$ in the ambient medium [of solar abundance] for the energy band below $100 \mathrm{keV}$, one may conclude that $n_{a} \cdot v_{\mathrm{s} 7}^{3} \cdot \theta^{2}>10^{5}$. That is a rather stringent requirement implying a high efficiency of electron acceleration by a relatively slow shock with $v_{\mathrm{s} 7} \gtrsim 1$ and $n_{a}>100 \mathrm{~cm}^{-3}$ for $\theta \sim 10$. A more detailed confrontation of the observational data and the model predictions, including $\mathrm{GeV} \gamma$-ray emission, will be described elsewhere.

An alternative interpretation of the observed hard X-ray sources could be a fast massive ballistically moving ejecta fragment interacting with a cloud. The fragment could drive a shock of velocity about $1000 \mathrm{~km} \mathrm{~s}^{-1}$ in the inter-clump medium providing a powerful source of X-ray emission both in continuum and lines (Bykov 2003). The fragments being enriched with metals (of an average nuclear charge $\langle Z\rangle$ ) have higher radiation efficiency, since $\eta \propto\langle Z\rangle$. The energetic problem of the bremsstrahlung emission models $\langle Z\rangle \cdot n_{a} \cdot v_{\mathrm{s} 7}^{3} \cdot \theta^{2}>10^{5}$ is alleviated. The line signature around $6.2 \mathrm{keV}$ required to fit the RXTE PCA data could be naturally explained in that case as a Fe $\mathrm{K}$ line complex predicted for the ejecta fragment model, though a contribution from hidden accreting sources (AGNs or $\mathrm{X}$-ray binaries) cannot be excluded.

A powerful wind of the early type O9V star HD 193322 located just $7^{\prime}$ from the NW bright clump (see Fig. 2) could also be considered as a candidate source for the observed emission. The star is the central object of the open cluster Collinder 419 located at an estimated distance $1.4 \mathrm{kpc}$ (e.g. McKibben et al. 1998) which is close to the $\gamma$-Cyg distance estimations. The trigonometric parallax of HD 193322 provided by the Hipparcos catalogue is $(2.1 \pm 0.61)$ mas marginally consistent with the distance. Interaction of the wind of HD 193322 with G78.2+2.1 would be a plausible source of particle acceleration and hard X-ray emission.
A deeper exposure of the field with ISGRI is required to detect (or to place meaningful upper limits) on the hard emission from the NW, NE, SE regions up to $120 \mathrm{keV}$. The presence of a few clumps of hard emission at a level above $10^{-11} \mathrm{erg} \mathrm{cm}^{-2} \mathrm{~s}^{-1}$ at the borders of $\gamma$-Cyg would support the hypothesis of SNR origin of the nonthermal clumps with important implications for particle acceleration mechanisms in SNRs.

Acknowledgements. The present work is partly based on observations with INTEGRAL, an ESA project with instruments and a science data centre funded by ESA member states (especially the PI countries: Denmark, France, Germany, Italy, Switzerland, Spain), Czech Republic and Poland, and with the participation of Russia and the USA. This research has made use of data obtained from the High Energy Astrophysics Science Archive Research Center (HEASARC), provided by NASA's Goddard Space Flight Center. We are grateful to F. Mavromatakis who generously provided us with optical data.

The work was partially supported by RFBR grants 03-02-17433, 0402-16595, 04-02-16042, RAS program, MK 2642.2003.02 and by the European Space Agency. R.A.C. was supported by NASA grant NNG04GA41G. G.R. and J.P.S. acknowledge support through the Belgian FNRS and the INTEGRAL PRODEX project. Support from the International Space Science Institute (Bern) through the international teams program is gratefully acknowledged.

\section{References}

Buckley, J. H., Akerlof, C. W., Carter-Lewis, D. A., et al. 1998, A\&A, 329,639

Bykov, A. M. 2003, A\&A, 410, L5

Bykov, A. M., Chevalier, R. A., Ellison, D. C., \& Uvarov, Yu. A. 2000, ApJ, 538, 203

Chevalier, R. A. 1999, ApJ, 511, 798

Esposito, J. A., Hunter, S. D., Kanbach, G., et al. 1996, ApJ, 461, 820

Gaisser, T., Protheroe, R., \& Stanev, T. 1998, ApJ, 492, 219

Goldwurm, A., David, P., Foschini, L., et al. 2003, A\&A, 411, L223

Higgs, L., Landecker, T., \& Roger, R. 1977, AJ, 82, 718

Landecker, T., Roger, R., \& Higgs, L. 1980, A\&AS, 39, 133

Lebrun, F., Leray, J. P., Lavocat, P., et al. 2003, A\&A, 411, L141

Lozinskaya, T. A. 1977, Sov. Astron. Lett., 3, 163

Lozinskaya, T. A., Pravdikova, V. V., \& Finoguenov, A. V. 2000, Astron. Lett., 26, 77

Lubinski, P. 2004 [arXiv: astro-ph/0405460]

Mavromatakis, F. 2003, A\&A, 408, 237

McKibben, W. P., Bagnuolo, W. G., Jr., Gies, D. R., et al. 1998, PASP, 110,900

Pannuti, T., Allen, G., Houck, J., et al. 2003, ApJ, 593, 377

Sturner, S. J., \& Dermer, C. D. 1995, A\&A, 293, L17

Sturner, S. J., Skibo, J. G., Dermer, C. D., \& Mattox, J. R. 1997, ApJ, 490, 619

Swanenburg, B. N., Bennett, K., Bignami, G. F., et al. 1981, ApJ, 243, L69

Uchiyama, Y., Takahashi, T., Aharonian, F. A., \& Mattox, J. R. 2002, ApJ, 571, 866

Winkler, C., Courvoisier, T., Di Cocco, G., et al. 2003, A\&A, 411, L1

Zhang, X., Zheng, Y., Landecker, T. L., \& Higgs, L. A. 1997, A\&A, 324,641 